\title{
Obesity and contraception: controversy?
}

This article was published in the following Dove Press journal:

Open Access Journal of Contraception

9 January 2012

Number of times this article has been viewed

\section{Rebecca D Goldberg Stefanie C Cardamone Amitasrigowri S Murthy \\ Department of Obstetrics and Gynecology, New York University School of Medicine, New York, NY, USA}

\begin{abstract}
Obesity is increasing worldwide and is affecting the reproductive health of women. Contraceptive considerations are difficult in obese women given concerns about efficacy and comorbid conditions. Once surgical treatment of obesity has occurred and weight loss initiated, fertility risks increase and unintended pregnancy can result; often at the time of greatest weight loss. Family planning counseling in the obese patient must account for both preoperative conditions as well as postoperative return to fertility.
\end{abstract}

Keywords: obesity, contraception, bariatric surgery

\section{Introduction}

Obesity and overweight are defined as "abnormal or excessive fat accumulation that may impair health."1 As per the Centers for Disease Control and Prevention (CDC), overweight and obesity are determined by using height and weight to calculate body mass index (BMI). Unhealthy ranges of weight, as measured by BMI, have been shown to increase the likelihood of certain health problems. ${ }^{2}$ There is, however, a distinct difference in measurement between overweight and obese. The World Health Organization (WHO), ${ }^{3}$ considers an adult with a BMI between 25 and 29.9 to be preobese with an increased risk of comorbidities, whereas an adult who has a BMI of 30 or higher is considered obese. WHO then divides obesity into three different classes according to BMI range: class I is BMI from 30 to 34.9, class II from 35 to 39.9, and class III over 40. The third class carries the highest risk of comorbidities. In 2006, the CDC showed that $34 \%$ of US adults aged 20 years and over were obese and this number has only increased since then. ${ }^{4}$

The purpose of this article is to review the prevalence of obesity in the world and examine its effects on fertility and contraceptive efficacy. After reviewing surgical treatments for obesity currently available, the article will focus on postoperative concerns of return of fertility and how the resulting anatomic changes affect contraceptive efficacy and choices.

\section{Obesity}

Obesity has more than doubled worldwide since 1980. In 2008, the WHO estimated that, globally, 1.5 billion adults aged 20 years or older were overweight. Of these, over 200 million men and nearly 300 million women were obese. ${ }^{5}$

In the USA, one-third of adults were obese in 2007-2008. ${ }^{5}$ In comparison with other countries, the USA carries the highest rate of obesity. The USA is followed by submit your manuscript $\mid$ www.dovepress.com

Dovepress

http://dx.doi.org/10.2147/OAJC.S17405
Open Access Journal of Contraception 2012:3 I-8

(C) 2012 Goldberg et al, publisher and licensee Dove Medical Press Ltd. This is an Open Access article which permits unrestricted noncommercial use, provided the original work is properly cited. 
Mexico, the UK, and Slovakia. Japan and South Korea have the lowest rates of obesity. ${ }^{1,5}$

Overweight and obesity are the fifth leading risk factor for global deaths. Almost 3 million adults die each year as a result of being obese or overweight. ${ }^{1}$ Raised BMI is a major risk factor for several diseases such as cardiovascular disease, type 2 diabetes, musculoskeletal disorders, coronary heart disease, hypertension, dyslipidemia, liver and gallbladder disease, sleep apnea and respiratory problems, as well as gynecological problems including abnormal menses, infertility, and some cancers. ${ }^{6}$ Obesity is an independent risk factor for the development of coronary artery disease in women. ${ }^{7}$ With every $10 \%$ increase in weight, blood pressure increases $6 \mathrm{mmHg}$ systolic and $4 \mathrm{mmHg}$ diastolic. ${ }^{8}$ Diabetes affects 246 million people worldwide, ${ }^{9}$ and the risk of developing type 2 diabetes increases as BMI increases. ${ }^{10}$ Obesity may lead to endometrial, breast, and colon cancers. Between $7 \%$ and $41 \%$ of the cancer burden is attributable to overweight and obesity. ${ }^{11}$

There are also several economic consequences of overweight and obesity. Direct medical costs may include preventative, diagnostic, and treatment services, whereas indirect costs relate to morbidity (the value of income lost from decreased productivity, restricted activity, bed days, and absenteeism) and mortality costs (the value of future income lost by premature death). In 2008, the medical care costs of obesity in the USA totaled about US\$147 billion. ${ }^{12}$ The cost of lost productivity due to obesity is approximately US $\$ 3.9$ billion, and another US\$33 billion is spent annually on weight-loss products and services. ${ }^{13}$ A study by Dor estimated that for women the individual annual cost associated with obesity (BMI of 30 or higher) was US\$4879. For men, the individual annual cost was calculated at US\$2646. Adding the value of lost life produces much higher annual costs of US\$8365 for obese women and US\$6518 for obese men. ${ }^{13}$

\section{Consequences of obesity regarding fertility}

Before pregnancy, obesity has a negative effect on fertility, primarily by reducing ovulation frequency. Ovulatory dysfunction may be caused by insulin resistance and the resulting hyperinsulinemia. ${ }^{14}$ High levels of insulin have a negative influence on the liver's production of sex hormone-binding globulin (SHBG), thereby leading to increases in androgen hormone levels. ${ }^{14,15}$ Increases in adipose tissue lead to chronically elevated levels of estrogen, from peripheral conversion of the high circulating androgens to estrogen. High insulin levels can cause augmentation of the ovarian response to luteinizing hormone $(\mathrm{LH})$ and thereby cause ovarian thecal cell production of androstenedione, which, along with the increased free testosterone from decreased SHBG, can impair normal ovulatory function. Normal ovulatory function can be further disrupted by leptin, secreted by adipocytes, whose main action is to decrease hunger and food intake. Leptin also causes inhibition of ovarian follicular development and steroidogenesis. ${ }^{14,15}$ Obese women have decreased fecundity, as evidenced by the decreased probability of natural conception in those with a BMI $>29 \mathrm{~kg} / \mathrm{m}^{2} .{ }^{14}$ The effects of obesity on fertility have been corroborated by data from the 2002 National Survey of Family Growth (NSFG). ${ }^{23}$ Higher rates of unintended pregnancy among obese women were expected, due to possible higher rates of contraceptive failure. However, the NSFG revealed no increased rate of unintended pregnancy among those who were obese and instead hinted at levels of subfertility, consistent with the theory of interference with normal ovulatory function. ${ }^{23}$

As the prevalence of obesity continues to increase worldwide, knowledge regarding the care of these women is becoming more necessary in the fields of obstetrics and gynecology. The high-risk nature of pregnancy in these women only reinforces the need for safe, effective contraception. A body of literature has emerged focusing on the unique metabolic changes observed in obesity, pharmacokinetic alterations, and their effect on contraceptive efficacy. ${ }^{20-22,24,25}$ Studies overall have been conflicting, but concern exists about increased rates of failure of hormonal contraceptives in this subset of women. The etiology of this finding is not well understood but is thought to be a result of alterations in the pharmacokinetics and metabolism of steroid hormone contraceptives. ${ }^{20-22,24,25}$

\section{Metabolism/Pharmacokinetics in obese women and contraception Normal metabolism}

Despite the increasing number of hormonal contraceptive options, most of these methods are a "variation on a theme." A basic understanding of the normal metabolism of hormonal contraceptive steroids is necessary to further define the changes seen in overweight and obese women.

Metabolism of oral hormonal contraceptive steroids starts with oral intake of the pill. After ingestion, dissolution occurs in the stomach and the steroid then goes to the intestine. Almost all of the available oral contraceptives contain ethinyl estradiol (EE) and one of 12 commonly used progestogens. ${ }^{20} \mathrm{EE}$ is absorbed from the stomach and upper intestine. The intestinal mucosa and gut flora then start the 
process of metabolism. Steroids are absorbed through the intestinal mucosa to the portal vein and, following this, the liver is reached. In the liver, further metabolism occurs via a number of metabolizing enzymes; this is referred to as hepatic first-pass metabolism. At this point, the progestins, which are prodrugs, then become metabolized to their active component. ${ }^{20}$ Once metabolized, steroids reach the systemic circulation. "Bioavailability" refers to the extent to which a steroid reaches the systemic circulation after first pass metabolism. However, with both EE and progestogens, there can be a wide variation in the bioavailability of the compounds. ${ }^{20}$ Unfortunately, there is no clear understanding of how the steroid levels in each individual woman could affect contraceptive efficacy and incidence of side effects. Nor is there information on how the combination of estrogen and progestin can affect the metabolism of each. ${ }^{20}$

\section{Pharmacokinetics}

"Pharmacokinetics" is defined as the ways in which the body transforms and utilizes a drug or medication. Pharmacokinetics is divided into four general areas: absorption, distribution, metabolism, and elimination. Each of these four processes is influenced by a number of factors including age, gender, coadministered drugs, alcohol intake, diet, as well as presence of infection. Obesity and body weight have also been proposed as other influential factors. ${ }^{21}$ Overall, the effects of obesity on the metabolism and the pharmacokinetics of hormonal contraceptives remain poorly understood and large, well-designed studies are lacking.

Most investigational studies of hormonal contraception have excluded women weighing over $130 \%$ of their ideal body weight. Moreover, little is understood regarding contraceptive safety and efficacy in obese women. With the goal of increased contraceptive efficacy in the obese population, studies have aimed at identifying the mechanism(s) of failure in this subset of women. It is known that the main mechanism of action of hormonal contraception involves the suppression of the hypothalamic-pituitary-ovarian axis. It has been hypothesized that pharmacokinetic alterations in obese women may lead to inadequate suppression of this system and thus allow ovulation and subsequently method failure. A study by Edelman et $\mathrm{al}^{21}$ of the pharmacokinetics of oral contraceptives in obese women used subjects placed on a combination monophasic birth control pill with $20 \mu \mathrm{g}$ EE/100 $\mu \mathrm{g}$ levonorgestrel (LNG) dosed on a cyclic schedule (21-days active pill with a 7-day hormone-free interval). The results of this study showed significant differences in LNG clearance, half-life, and time to reach steady state between obese and normal-BMI women. The half-life in obese subjects was twice that of normal-BMI subjects. Thus, the time to reach steady state was also doubled in obese women in comparison with those women with a normal BMI. These results suggest that obese women might require up to 5 days longer for steady-state inhibitory drug concentrations to be reached. This creates a longer time period during which a pregnancy might occur. ${ }^{21}$

Another study demonstrated lower serum concentrations of both EE and LNG in obese women, but trough levels were not significantly different. ${ }^{22}$ The implication of these findings on the contraceptive efficacy of oral contraceptives may be small or negligible, as the minimum concentrations of LNG were sufficient to suppress ovulation. Again, there was evidence of more follicular activity in obese patients. Although not statistically significant, there was a trend toward larger follicular size with sonographic imaging as well as higher estradiol concentrations in obese subjects than in normal weight subjects. ${ }^{22}$

There is less data about the pharmacokinetics of non-oral routes. Only one study looked at injectable contraception in obese women. ${ }^{19}$ The purpose was to evaluate the effect of obesity on follicular development in those using subcutaneous depot medroxyprogesterone acetate (DMPA) (DMPA-SC 104 mg; Pfizer, Inc, New York, NY) as contraception. Large intersubject variability was noted in the serum levels of DMPA. All levels were above that needed to inhibit ovulation but lower levels were noted in obese subjects. ${ }^{32}$

\section{Obesity and contraceptive efficacy Combined hormonal regimens}

Combined hormonal contraceptives containing both a synthetic estrogen component as well as a progestin component derive their contraceptive efficacy from the effects of progestin. The progestin component acts primarily through the suppression of ovulation via a negative feedback mechanism. The pulsatile frequency of gonadotropin-releasing hormone release is decreased; thereby decreasing the amount of both follicle-stimulating hormone (FSH) and LH. ${ }^{15}$ Decreased levels of FSH inhibit follicular development thus preventing estradiol production. The absence of mid-cycle LH surge and the following inhibition of follicular development prevent ovulation. Secondary progestational effects include increased viscosity of cervical mucus and endometrial changes, which may decrease the likelihood of implantation. ${ }^{15}$

It was described in the 2002 NSFG that obese women were more likely to have a contraceptive failure while using an oral contraceptive. ${ }^{23}$ Hispanic or black women were more 
likely to experience these failures and, as already noted, were also more likely to be overweight or obese. ${ }^{23}$ Holt et al were among the first to raise concerns about contraceptive pill failure being increased in obese women. ${ }^{24}$ This group noted increased pill failure in those weighing $>70 \mathrm{~kg}$. This held true despite the estrogen dose in the oral contraceptive and in both a retrospective and prospective study. ${ }^{24,25}$ This finding is in direct contrast to other data, which have not found an increased risk of pill failure in obese women. Trussell et al reviewed studies examining contraceptive efficacy in obese women; the authors found that obesity conferred no increased risk of failure. ${ }^{26}$ Two of the eight studies were prospective clinical trials and had measured body weights, thereby confirming that there was no increased risk of failure with oral contraceptive use in women either overweight or obese.

In obese women, minimal data exist when looking at contraceptive efficacy with non-oral methods. For both the contraceptive patch (Ortho Evra/Evra, Ortho-McNeil, Raritan, NJ) and the vaginal ring (NuvaRing, ScheringPlough, Kenilworth, NJ), there are no data on women over $130 \%$ of ideal body weight. In the analysis of pooled data from the contraceptive patch approval studies $(n=3319)$, there were 15 pregnancies; five pregnancies were noted to be in women weighing $>90 \mathrm{~kg} .{ }^{27}$

No prospective studies have examined vaginal ring use in those who are either overweight or obese; none of the preapproval studies included women weighing $>80 \mathrm{~kg}$. Efficacy is thought to be similar in obese and normal weight women due to local absorption of medication through the vaginal mucosa. ${ }^{28}$ A reanalysis of Phase III data for the ring was performed and no pregnancies were noted in heavier women $(n=6047$; weight range, $88-272 \mathrm{lbs}) .{ }^{29}$ Given that the vaginal delivery of hormones in the ring bypasses first-pass metabolism by the liver, hormone levels may be higher in obese women, consequently allowing for improved hypothalamic pituitary ovarian inhibition.

Cycle control with combined hormonal contraceptive use in obese women is unknown. However, a retrospective analysis of 2893 women (613 of whom were overweight or obese) using two different low-dose oral contraceptive formulations found no difference in breakthrough bleeding or spotting. ${ }^{30}$ It can only be presumed that cycle control in obese women using non-oral preparations is equally efficacious.

\section{Progestin-only methods \\ Progestin-only pills}

Little data exist to support any evidence of decreased efficacy of progestin-only pills in obese or overweight women.
A recent European study on oral contraceptive surveillance monitored more than 50,000 oral contraceptive users and found no association between obesity and progestin-only pills. ${ }^{31}$ The number of obese women included in this study, however, is unknown.

\section{Contraceptive injection}

The efficacy of the DMPA (150 mg; Depo-Provera, Pfizer, Inc) injection has been shown to be unaffected by weight, although concerns about changes in weight seen with this method of contraception are well known. The most common reason for discontinuation of this method of contraception is weight gain among users. ${ }^{17}$ The etiology of weight gain in DMPA users remains undefined, but increased serum lipids, fluid retention, and fat mass deposition have been proposed. Retrospective studies investigating weight variations in DMPA users and those using other contraceptive methods have been conflicting. Some have failed to control for baseline weight and BMI. In counseling the obese population, it is important to ensure understanding of how DMPA will affect their weight. A retrospective cohort study compared 379 DMPA users to 1074 TCu380A intrauterine-device (IUD) users over a similar period of time. ${ }^{17}$ Women were allocated to three groups based on baseline BMI (normal weight, overweight, obese). At the end of 3 years of use, the DMPA users had a significantly higher weight gain than nonusers. A progressively larger weight gain was noted with increasing duration of use. Interestingly, changes in weight were less pronounced in the third group of women, which included those with baseline obesity. BMI increased in $23.7 \%$ of DMPA users versus $11.7 \%$ of IUD users. The authors speculate that DMPA use may trigger a metabolic alteration in normal and overweight women, which results in a gradual increase in weight. ${ }^{17}$ A number of other studies have been done on weight gain with DMPA use, with most reporting a weight gain of approximately $4 \mathrm{~kg}$ in users. ${ }^{19,32}$ However, findings have been conflicting and well-executed, prospective, controlled studies are lacking.

Reasonably, providers may be reluctant to recommend DMPA to obese women as it could result in further weight increase. DMPA, however, has certain benefits compared with other forms of hormonal contraception, including unaltered efficacy. Nevertheless, while DMPA was initially thought not to increase the risk of thromboembolism, a particular concern in obese women, recent data suggests that it may cause a threefold increase in risk of venous thromboembolism (VTE) even when controlled for BMI. 
This finding may also make providers more reluctant to use DMPA in obese women. ${ }^{33}$

\section{Contraceptive implant}

The preclinical trials for the etonogestrel-containing implant, known as Implanon (Merck, Kenilworth, NJ), included only 134 women who weighed $>70 \mathrm{~kg}$ and no pregnancies were noted in this group. ${ }^{18}$ Product labeling encourages clinical judgment regarding the 3-year limit when using in obese women. No definitive statement on efficacy can be made when looking at use in obese women. No changes in the pattern of irregular bleeding based on weight were noted in Implanon users, although this finding cannot be verified. ${ }^{18}$

\section{Obesity treatments}

Bariatric surgical procedures currently used can be classified as either restrictive or malabsorptive procedures. Restrictive procedures include vertical banding gastroplasty, adjustable gastric banding, and intragastric balloon. ${ }^{34}$ These procedures involve the creation of a small gastric pouch leading to early satiety. Malabsorptive procedures, including the Roux-en-Y gastric bypass, biliopancreatic diversion, and duodenal switch, involve bypassing a large section of small bowel thus decreasing potential for absorption. These procedures can be performed via laparotomy or laparoscopy depending on surgeon expertise, with laparoscopic techniques being used more often. Gastric bypass procedures and gastric banding are currently the most common bariatric procedures performed. ${ }^{34}$ In general, laparoscopic approaches have been shown to have equal efficacy with the benefit of fewer postoperative complications such as wound infections, smaller incisions, faster recovery, and earlier ambulation with fewer respiratory complications. ${ }^{35}$

Currently, indications for bariatric weight-loss procedures include patients with a BMI $>40 \mathrm{~kg} / \mathrm{m}^{2}$ or $>35 \mathrm{~kg} / \mathrm{m}^{2}$ with coexisting conditions such as severe sleep apnea, diabetes, cardiopulmonary disease, or joint disease who have failed with nonsurgical weight-loss methods including diet, exercise, behavioral modification, and psychological support. ${ }^{36}$ Studies have confirmed the multitude of benefits of bariatric surgery including improvements in glucose intolerance, hypertension, hyperlipidemia, sleep apnea, gastric reflux disease, joint disease, urinary incontinence, and other diseases. ${ }^{37}$ Major complications include ventral hernia, gallstone disease, stricture, band slippage, pouch enlargement, small-bowel obstruction, band erosion, ulceration, leaks, wound infections, stomal stenosis, staple-line failure, severe esophageal dilation, vomiting, diarrhea, bleeding, vitamin deficiencies, splenic injury, fascial dehiscence, and pulmonary embolism. Overall mortality is low, estimated to be $<1 \%$. Long-term complications include dumping syndrome, cholelithiasis, and nutritional deficiencies. Malabsorptive procedures are more likely to cause the latter and patients are required to take nutritional supplements postoperatively. ${ }^{36,37}$

\section{Changes in health after surgery}

Altered reproductive hormone profiles have been noted in those undergoing bariatric surgery. Postoperative improvements in both obesity-related illnesses as well as fertility have been reported. Two reviews of patients undergoing bariatric surgery noted either resolution or improvement of type 2 diabetes mellitus. ${ }^{38-40}$ Weight loss was also associated with a reduction in cholesterol and triglyceride levels as well as normalization of blood pressure and obstructive sleep apnea. ${ }^{39}$ Given the improvements seen in both diabetes and hypertension, one can presume that similar resolution of hormonal milieu occurs with the reproductive hormones, thereby causing a return to fertility. This underscores the need for effective contraception during the highest weight-loss period. In the postoperative setting, patients are counseled to avoid pregnancy, but little data exist on the methods of contraception women use during the first year after surgery. One self-administered survey of 45 postoperative women showed that $97 \%$ knew to avoid pregnancy for 1 year and $90 \%$ used contraception during this time. One-third of women used a highly effective method of birth control and $37 \%$ used only condoms. In contrast, if women obtained their contraception from an obstetrician/gynecologist, then all methods of contraception were highly effective..$^{41,42}$

\section{Return of fertility after surgery}

Since fertility may increase after bariatric surgery, after weightloss surgery women are in need of reliable contraception. It has been reported that approximately $10 \%$ of women aged $<40$ years will become pregnant within 3 years of bariatric surgery. ${ }^{38-43}$ The literature on pregnancy outcomes and complications in women who have undergone weight-loss surgery is limited and conflicting. ${ }^{43}$ Generally, it is recommended that women defer pregnancy until at least 1 to 2 years postoperatively, corresponding to the period when the most rapid weight loss occurs. ${ }^{40}$

Interestingly, the altered hormonal profile seen in obese women appears to normalize after weight-loss surgery. ${ }^{39,44}$ A number of studies have found an increase in SHBG, FSH, and LH, as well as decreased estradiol and testosterone levels after weight-loss surgery in obese women. ${ }^{45}$ With a decrease 
in adipose tissue, there is a decrease in circulating estradiol. A lower level of estradiol also decreases negative feedback at the level of the hypothalamus, resulting in increased secretion of gonadotropin-releasing hormone and subsequently LH and FSH. Concurrently, postoperative normalization of menstrual irregularities has been reported along with an associated increase in fertility. ${ }^{44} \mathrm{~A}$ study by Rochester et al ${ }^{38}$ evaluated daily urinary hormone excretion over the course of one menstrual cycle in normal weight women as well as obese women before and 6 months after surgical weightloss surgery. All patients lost at least $25 \%$ of initial body weight. The study suggested at least partial recovery of luteal function, with a more than twofold increase in pregnanediol glucuronide and increase in urinary LH. Urinary LH levels reached that of normal weight women. ${ }^{38,39}$

\section{Contraception after bariatric surgery}

Overall, the data available on the efficacy and safety of contraception in women with a history of bariatric surgery are poor. ${ }^{40}$ Concerns have been raised about the efficacy of oral contraceptives after bariatric surgery. Studies comparing obese women after gastric bypass with controls have demonstrated lower levels of circulating contraceptive hormones. ${ }^{39}$ This finding is thought to be secondary to decreased absorption of constituent hormones due to the surgical bypass of a large area of stomach and duodenum, as well as long-term postoperative diarrhea and vomiting. What follows is that contraceptive failure is more likely in women who undergo malabsorptive bariatric procedures. Another study demonstrated contraceptive failure only in patients using oral contraceptives as the sole form of contraception postoperatively, suggesting that oral contraceptives as the only form of contraception in this population of patients should be avoided. ${ }^{46}$

Pharmacokinetic studies on women after gastric bypass have confirmed lower levels of circulating levels of contraceptive hormones. Victor et al demonstrated that postoperative patients had lower levels of LNG at 2, 4, and 6 hours after ingestion. ${ }^{44}$ However, baseline levels were still sufficient for contraception. Given the conflicting evidence in the literature, it should generally be noted that surgical procedures resulting in malabsorption have the potential to decrease oral contraceptive efficacy. Even less is understood about the effects of restrictive procedures that are becoming more common in current practice.

The study by Gerrits et al, ${ }^{40}$ which evaluated 40 obese women, looked primarily at oral contraceptives after bariatric surgery. In this series, 2 out of 9 women became pregnant during 2 years of follow-up (22\%). The study noted that none of the IUD or DMPA users became pregnant, suggesting that the efficacy of these contraceptives is not decreased after weight-loss surgery. ${ }^{40}$

Safety concerns have also been raised, including increased risks of thromboembolism with the use of combined oral contraceptives, as well as accelerated bone loss with the use of DMPA in patients with already depleted bone reserve secondary to malabsorption postoperatively. ${ }^{47}$ Obesity is considered an independent risk factor for the development of VTE. The presumptive etiology is from an increase in procoagulant factors like Factor VII, Factor VIII, Factor XII in addition to fibrinogen. ${ }^{48,49}$ An additive risk with venous stasis also occurs in obesity. Most reviews have found an additive increase in risk for VTE in obese women. When combined with oral contraceptives, this risk is increased even further. One study comparing obese to non-obese controls found that obese subjects have twice the risk of VTE and obese subjects on oral contraceptives had a tenfold increased risk. ${ }^{48}$ Generally, the use of hormonal contraception, including oral combined oral contraceptives, patch, or the vaginal ring is accepted in obese women. A BMI of $>30 \mathrm{~kg} / \mathrm{m}^{2}$ is a Category 2 condition for all combined hormonal methods. These methods can generally be used but more careful follow-up may be required.

Bone loss frequently occurs after bariatric surgery because of vitamin deficiencies, particularly with malabsorptive procedures. Studies have also demonstrated increased bone turnover in this setting. ${ }^{47}$ The clinical implications of these bone changes are unknown and it is unknown if the use of DMPA in these patients has any substantial further detrimental effect on bone health.

\section{Contraception in obese women}

The literature suggests that obese women are less likely to use contraception. A study evaluating a population of obese women prior to bariatric surgery reported that $51.8 \%$ of women reported use of contraception in the previous 12 months. ${ }^{50}$ A majority (74.3\%) of these women reported using contraception "at all times," while an additional $10.5 \%$ reported use "most of the time." The most common forms of contraception used in this population of women were condoms and oral contraceptives. ${ }^{41,50,51}$

\section{Conclusion}

The obesity epidemic underscores the need for effective contraception. Conflicting data exist regarding the efficacy of oral contraceptives in obese women, although most 
demonstrate no decrease in efficacy. Conversely, the sparse existing data on contraceptive efficacy of non-oral combined hormonal methods suggest that local delivery of hormones as by the vaginal ring, for example - provides high levels of efficacy, but that the contraceptive patch requires monitoring of body weight before initiation. However, long-acting reversible methods such as IUDs appear to demonstrate very high levels of efficacy due to the immediate local contraceptive effect, while avoiding both the increased risk of VTE as well as increasing vascular risks with other comorbid conditions. In those women who go on to receive surgical treatment for obesity, it is unclear if continuing hormonal contraceptives would be a safe and effective choice given the digestive changes occurring after surgery. Again, in these patients, a long-acting reversible method such as an IUD would be an ideal choice to prevent pregnancy in the immediate postsurgical period that incorporates rapid weight loss. Family planning counseling for obese patients needs to consider the risks caused by comorbid conditions of obesity as well as theoretical concern for decreased efficacy. In those obese patients deciding to move on to surgical treatments, family planning counseling must consider the preoperative risks derived from obesity as well as the comorbid conditions and also the postoperative risk of increased fertility. It is most distressing that, despite knowledge of the need to avoid pregnancy during the time of rapid weight loss, most postoperative women of reproductive age are not using a highly effective form of contraception. Special attention needs to be paid to this time of increased risk of pregnancy.

\section{Disclosure}

The authors declare no conflicts of interest in this work.

\section{References}

1. World Health Organization (WHO). Obesity and overweight. Factsheet No 311. Geneva: WHO; 2011. Available from: http://www.who.int/ mediacentre/factsheets/fs311/en/. Accessed April 3, 2011.

2. Centers for Disease Control and Prevention (CDC) Defining overweight and obesity [web page on the Internet]. Atlanta, GA: CDC; 2010 [updated June 21, 2010]. Available from: http://www.cdc.gov/obesity/defining. html\#. Accessed April 3, 2011.

3. The Global Challenge of Obesity and the International Obesity Task Force: tables. Los Angeles, CA: International Union of Nutritional Sciences; nd. Available from: http://www.iuns.org/features/obesity/tabfig. htm\#Figure\%201. Accessed August 26, 2011.

4. CDC. Overweight and obesity: data and statistics [web page on the Internet]. Atlanta, GA: CDC; 2011 [updated May 16, 2011]. Available from: http://www.cdc.gov/obesity/data/index.html. Accessed June 8, 2011.

5. CDC. Overweight and obesity: economic consequences [web page on the Internet]. Atlanta, GA: CDC; 2011 [updated March 28, 2011]. Available from: http://www.cdc.gov/obesity/causes/economics.html. Accessed April 3, 2011.
6. National Diabetes Information Clearinghouse. National diabetes statistics, 2011: fast facts on diabetes [web page on the Internet]. Bethesda, MD: National Diabetes Information Clearinghouse; 2011. Available from: http://diabetes.niddk.nih.gov/dm/pubs/statistics/\#fast. Accessed June 8, 2011.

7. Kulie T, Slattengren A, Redmer J, Counts H, Eglash A, Schrager S. Obesity and women's health: an evidence-based review. J Am Board Fam Med. 2011;24(1):75-85.

8. Murthy AS. Obesity and contraception: emerging issues. Sem Reprod Med. 2010;28(2):156-163.

9. BD - Worldwide. Newsroom: diabetes care [web page on the Internet]. Franklin Lakes, NJ: Becton, Dickinson and Company; 2011. Available from: http://www.bd.com/press/newsroom/diabetes.asp. Accessed April 3, 2011.

10. Packinathan I, Finer N. Medical consequences of obesity. Obesity. 2003:8-12.

11. Bianchini F, Kaaks R, Vainio H. Overweight, obesity and cancer risk. Lancet Oncol. 2002;3:565-574.

12. Mansour D. Implications of the growing obesity epidemic on contraception and reproductive health. J Fam Plan Reprod Health Care. 2004;30(4):209-211.

13. Avi Dor CF. Heavy Burden Report. Washington DC: The George Washington University, School of Public Health and Health Services, Department of Health Policy; 2010. Available from: http://www.gwumc. edu/sphhs/departments/healthpolicy/pdf/HeavyBurdenReport.pdf. Accessed April 3, 2011.

14. Wuntakal R, Hollingworth T. The implications of obesity on pregnancy. Obstet Gynecol Reprod Med. 2009;19(12):344-359.

15. Speroff L, Fritz MA. Clinical Gynecologic Endocrinology and Infertility. 7th ed. Philadelphia, PA: Lippincott Williams and Wilkins; 2005:200-214.

16. Kaneshiro B, Edeman A, Carlson N, Nichols M, Jensen J. The relationship between body mass index and unintended pregnancy; results from the 2002 National Survey of Family Growth. Contraception. 2008;77(4):234-238.

17. Pantoja M, Madeiros T, Baccarin MC, Morais SS, Bahamondes L, Fernandes AM. Variations in body mass index of users of depotmedroxyprogesterone acetate as a contraceptive. Contraception. 2010;81(2):107-111.

18. Graesslin O, Korver T. The contraceptive efficacy of Implanon: a review of clinical trials and marketing experience. Eur J Contracept Reprod Health Care. 2008;13(Suppl 1):4-12.

19. Kaunitz AM, Darney PD, Ross D, Wolter KD, Speroff L. Subcutaneous DMPA vs intramuscular DMPA: a 2 years study of contraceptive efficacy and bone mineral density. Contraception. 2009;80(1):7-17.

20. Edelman AB, Cherala G, Stancyzk FZ. Metabolism and pharmacokinetics of contraceptive steroids in obese women: a review. Contraception. 2010;82:314-323.

21. Edelman AB, Carlson NE, Cherala G, et al. Impact of obesity on oral contraceptive pharmacokinetics and hypothalamic-pituitary-ovarian activity. Contraception. 2009;80:119-127.

22. Westoff C, Torgal, A, Mayeda E, Pike, M, Stanczyk, F. Pharmacokinetics of a combined oral contraceptive in obese and normal-weight women. Contraception. 2010;81:474-480.

23. Brunner Huber LR, Toth JL. Obesity and oral contraceptive failure: Findings from the 2002 National Survey of Family Growth. Am J Epidem. 2007;166(11):1306-1311.

24. Holt V, Cushing Haugen K, Daline J. Body weight and risk of oral contraceptive failure. Obstet Gynecol. 2002;99:820-827.

25. Holt V, Scholes D, Wicklund K, Cushing-Haughen K, Daling J. Body mass index, weight and oral contraceptive failure risk. Obstet Gynecol. 2005; 105:46-52.

26. Trussel J, Schwarz EB, Guthrie K. Obesity and oral contraceptive pill failure. Contraception. 2009;79(5):334-338.

27. Zieman M, Guillebaud J, Weisberg E, Shangold GA, Fisher AC, Creasy GW. Contraceptive efficacy and cycle control with the Ortho Evra/Evra transdermal system: the analysis of pooled data. Fertil Steril. 2002;77(2 Suppl 2):S13-S18. 
28. Dieben TO, Roumen FJ, Apter D. Efficacy, cycle control, and user acceptability of a novel combined contraceptive vaginal ring. Obstet Gynecol. 2002;100(3):585-593.

29. Westhoff C. Higher body weight does not affect NuvaRing's efficacy [abstract]. Obstet Gyneco.l 2005;105(Suppl 4):56S.

30. Bjarnadottir RI, Tuppurainen M, Killick SR. Comparison of cycle control with a combined contraceptive vaginal ring and oral levonorgestrel/ ethinyl estradiol. Am J Obstet Gynecol. 2002;186(3):389-395.

31. Dinger J, Cronin M, Mohner S, Schnellschmimidt I, Minh T, Westhoff C. Oral contraceptive effectiveness according to body mass index, weight, age and other factors. (EURAS-OC). Am JObstet Gynecol. 2009;80(1):7-17.

32. Segall-Gutierrez P, Taylor D, Liu X, Stanczyk FZ, Azen S, Mishell DR. Follicular development and ovulation in extremely obese women receiving depo-medroxyprogesterone acetate subcutaneously. Contraception. 2010;81:487-495.

33. van Hylckama Hlieg A, Helmerhost FM, Rosendall FR. The risk of deep venous thrombosis associated with injectable depotmedroxyprogesterone acetate contraceptives or a levonorgestrel intrauterine device. Arterioscler Thromb Vasc Biol. 2010;30:2297-2300.

34. Tice JA, Karliner L, Walsh J, Peterson AJ, Feldman MD. Gastric banding or bypass? A systematic review comparing the two most popular bariatric procedures. Amer J Med. 2008;121:885-893.

35. Schauer PR, Ikramuddin S, Gourash W, Ramanathan R, Luektich J. Outcomes after laparoscopic Roux-en-Y gastric bypass for morbid obesity. Ann Surg. 2000;232(4):515-529.

36. DeMaria EJ, Sugerman HJ, Kellum JM, Meador JG, Wolfe LG. Results of 281 consecutive total laparoscopic Roux-en-Y gastric bypasses to treat morbid obesity. Ann Surg. 2002;235(5):640-645.

37. Hall JC, Watts JM, O'Brien PE, et al. Gastric surgery for morbid obesity. The Adelaide Study. Ann Surg.1990;211:419-427.

38. Rochester D, Jain A, Polotsky AJ, Polotsky H, Gibbs K, Isaac B, et al. Partial recovery of luteal function after bariatric surgery in obese women. Fertil Steril. 2009;92:1410-1415.

39. Mehri Z. Impact of bariatric surgery on female reproduction. Fertil Steril. 2009;92(5):1501-1508
40. Gerrits EG, Ceulemans R, van Hee R, Hendrickx L, Totte E. Contraceptive treatment after biliopancreatic diversion needs consensus. Obesity Surg. 2003;13:378-382.

41. Krishnan S, Hacker M, Haider S, et al. Contraceptive counseling and utilization in women who underwent bariatric surgery. Poster presented at Annual Meeting of the Association of Reproductive Health Professionals, October 1, 2009, Los Angeles, CA.

42. Elkins G, Whitfield P, Marcus J, Symmonds R, Rodriguez J, Cook T. Noncompliance with behavioral recommendations following bariatric surgery. Obesity Surg. 2005;15:546-551.

43. Maggard MA, Yermilov I, Li Z, et al. Pregnancy and fertility following bariatric surgery. A systematic review. JAMA. 2008;300(19): 2286-2296.

44. Victor A, Odlind V, Kral JG. Oral contraceptive absorption and sex hormone binding globulins in obese women: effects of jejunoileal bypass. Gastroenterol Clin North Am. 1987;16:483-491.

45. American Society for Reproductive Medicine. Obesity and reproduction: an educational bulletin. Practice Committee Birmingham, AL. Fertil Steril. 2008;90(Suppl 3):S21-S29.

46. Merhi ZO. Challenging oral contraception after weight loss by bariatric surgery. Gynecol Obstet Invest. 2007;64:100-102.

47. Viegas M, Vasconselos RS, Neves AP, Diniz ET, Bandeira F. Bariatric surgery and bone metabolism: a systematic review. Arq Bras Endocrinol Metabol. 2010;54(2):158-162.

48. Abdollahi M, Cushman M, Rosendaal FR. Obesity: risk of venous thrombosis and the interaction with coagulation factor levels and contraceptive use. Thromb Haemost. 2003;89(3):493-398.

49. Pomp ER, le Cessie S, Rosendaal FE, Doggen CJ. Risk of venous thrombosis: obesity and its joint effect with oral contraceptive use and prothrombotic mutations. Br J Haematol. 2007;139:289-296.

50. Gosman G, King W, Schrope B, Steffen K, Strain G, et al. Reproductive health of women electing bariatric surgery. Fertil Steril. 2010;94: 1426-1431.

51. Paulen ME, Zapata LB, Cansino C, Curtis KM, Jamieson D. Contraceptive use among women with a history of bariatric surgery: a systematic review. Contraception. 2010;82:86-94.
Open Access Journal of Contraception

\section{Publish your work in this journal}

Open Access Journal of Contraception is an international, peerreviewed, open access, online journal, publishing original research, reports, reviews and commentaries on all areas of contraception. In addition to clinical research, demographics and health-related aspects, the journal welcomes new findings in animal and preclinical studies

\section{Dovepress}

relating to understanding the biological mechanisms and practical development of new contraceptive agents. The manuscript management system is completely online and includes a very quick and fair peer-review system. Visit http://www.dovepress.com/testimonials.php to read real quotes from published authors. 